\title{
INNOVACIÓN EN EL GERENCIAMIENTO DE LA SEGURIDAD Y SALUD OCUPACIONAL POST COVID-19
}

\author{
Guillermo Francisco Barrera Córdova \\ Ingeniero Industrial y Máster en Prevención de Riesgos Ocupacionales, Perito en áreas especializadas acreditado \\ por el Ministerio de Trabajo y Previsión Social de El Salvador (PSO-16-19), Catedrático de programas de formación \\ continua y de la Maestría en Recursos Humanos de la Universidad Evangélica de El Salvador \\ guibarrera.sv@gmail.com ORCID https://orcid.org/0000-0002-2929-7667
}

Recibido: 25 de enero de 2021 / Aceptado: 18 de mayo de 2021

\section{RESUMEN}

En este artículo se hace una descripción de la evolución que el gerenciamiento de la Seguridad y Salud Ocupacional han tenido en los últimos meses producto de la pandemia por el virus SARS-CoV-2. Asimismo, se hace un abordaje al hecho de como la innovación es un factor importante por considerar para que el gerenciamiento de la seguridad sea efectivo en las operaciones de cualquier organización independiente al rubro que pertenezca. Es importante tomar en cuenta que las brechas generacionales, personas «vulnerables» y demás personas con alto riesgo que una vez contraído el virus puede afectar de manera grave la salud de ellas son factores por considerar al momento de gerenciar la Seguridad y Salud en el Trabajo, con el fin de garantizar la continuidad del negocio.

Palabras clave: COVID-19, gerencia, seguridad, laboral, salud, ocupacional, innovación, El Salvador. 


\title{
INNOVATION IN OCCUPATIONAL HEALTH AND SAFETY MANAGEMENT POST COVID-19
}

\begin{abstract}
This article describes the evolution that Occupational Health and Safety management has had in recent months as a result of the SARS-CoV-2 virus pandemic, as well as an approach to the fact of how innovation It is an important factor to be considered for the Safety Management to be effective in the operations of any organization independent of the category it belongs to. It is important to take into account that generation gaps, "vulnerable" people and other people at high risk who, once contracted by the virus, can seriously affect their health are factors to consider when managing Safety and Health at Work, in order to guarantee business continuity.
\end{abstract}

Key words: COVID-19, managment, safety, labour, health, innovation, El Salvador.

\section{Introducción}

Todos deseamos un lugar de trabajo seguro y saludable, pero lo que estamos dispuestos a realizar para lograr este objetivo puede variar de persona a persona. El gerenciar es trabajar con las virtudes de otros para alcanzar los objetivos de la organización. En la medida que la gerencia adquiere un nivel más alto desde el punto de vista jerárquico, se espera un mayor grado de responsabilidad.

En ese sentido, al gerenciar la Seguridad y Salud en el Trabajo (SST) es un reto para toda organización; sobre todo, considerando que desde el año 2010 en El Salvador con la entrada en vigor de la Ley de Prevención de Riesgos en los Lugares de Trabajo, se ha «potencializado» el tema de seguridad y salud ocupacional hasta un cierto nivel de compromiso.

Lastimosamente el tema de seguridad y salud ocupacional en la mayoría de las empresas privadas en El Salvador se ha visto como un «mal necesario», pocas veces le han dado la importancia que esta área requiere, considerando que, de una persona, por lo general nombrado como «encargado de seguridad» es quien marca el tono del programa de seguridad y salud en la compañía, teniendo en sus hombros una gran responsabilidad. Sin embargo, la misma legislación salvadoreña no considera la figura 
de un encargado ni mucho menos a un Gerente de Seguridad o Líder como alguien indispensable para la administración de esta área tan sensible e importante para la continuidad de un negocio.

Teniendo en cuenta estos antecedentes de la realidad de la Seguridad y Salud Ocupacional en El Salvador y, sobre todo, la manera en cómo la Empresa Privada ha visto este tema en sus operaciones; es en el año 2020 a raíz de la pandemia de COVID-19, la cual ha puesto en jaque la economía mundial y ha generado muchas dificultades y, sobre todo, cambios culturales en las organizaciones y en la población en general, el interés por fortalecer la temática relacionada con la Seguridad y Salud Ocupacional ha tomado un giro de ciento ochenta grados y un auge que en todo lugar de trabajo se habla de medidas de prevención de riesgos.

Por lo que el reto de las organizaciones que quieran salir adelante en esta nueva realidad es entender que hoy en día innovar la manera de como gerenciar la Seguridad y Salud en el Trabajo es la clave para el éxito de las operaciones de las Empresas Privadas en El Salvador y lo más importante garantizar la continuidad del negocio.

Mientras el «encargado de seguridad» siga siendo un simple encargado o los Comités de Seguridad sean las figuras responsables de implementar las medidas preventivas en las organizaciones no podemos esperar resultados diferentes adaptables a esta nueva realidad operativa en las organizaciones.
El ámbito de estudio de la presente investigación será en la región metropolitana del gran San Salvador, marco geográfico donde se concentran la mayor parte de empresas del sector privado, y el período de desarrollo de la investigación se estima en un lapso de 10 semanas.

\section{Metodología}

La metodología utilizada conforme al propósito de investigación y análisis del objeto de estudio, resultó conveniente el uso de la técnica de la observación documental, para conocer los principales enfoques, definiciones y teorías relacionadas con las temáticas de la innovación, el gerenciamiento y el COVID-19.

Con el fin de realizar una integración entre estas tres vertientes que permitirán conocer como las tres se interrelacionan para generar el cambio cultural y el enfoque administrativo que hoy en día las empresas deben implementar para hacerle frente a esta nueva normalidad.

\section{Marco teórico conceptual}

\section{¿Qué es la innovación?}

La innovación no solo se trata de laboratorios de investigación y desarrollo con fuerte financiamiento, ejércitos de científicos y listas de patentes; más bien, el concepto de innovación debe ampliarse para incluir un nuevo pensamiento radical en todo el espectro, procesos, productos, tecnologías, servicios. 
El éxito y la sobrevivencia en esta nueva realidad hacen que las empresas necesiten más que buenas ideas y capacidad de ejecutarlas con excelencia. Desarrollar una capacidad sostenible de innovación continua tiene que ser forzosamente un proceso liderado al más alto nivel de la Empresa (INCAE Bussiness School, 2016).

«La innovación es el medio con el cual pueden explotar el cambio como una oportunidad de mejora alternativa». (Drucker, 1985)

«La innovación como la introducción de un nuevo o significativamente mejorado producto (bien o servicio), de un proceso, de un nuevo método organizativo en las prácticas internas de la empresa, la organización del lugar de trabajo o las relaciones exteriores» (Echavarría, 2008).

La innovación en materia de prevención de riesgos es un elemento clave y diferenciador que permite aumentar la competitividad de las empresas, mejorar su calidad de vida y garantizar la continuidad del negocio. La inno- vación, por lo tanto, debe estar muy presente al momento de gerenciar la seguridad y salud en el trabajo. Sin embargo, cuando se habla de innovación es de reconocer que, en El Salvador, poco o nada se hace por impulsar una cultura de innovación y mucho menos asociar este tema a la seguridad y salud en el trabajo.

En el Índice de Competitividad Global 2019 del Foro Económico Mundial, El Salvador cayó cinco posiciones (El Salvador.com, 2019). La escasa capacidad de innovación que tiene El Salvador se ha convertido en una de las mayores anclas de peso que no le permiten a la economía local ser más competitiva, según el último Reporte Global de Competitividad elaborado por el Foro Económico Mundial (FEM); según la Figura 1, podemos ver que El Salvador se encuentra ranqueado en la posición 103, solo por encima de países como Nicaragua, Venezuela e incluso Haití, superado por escasos puntos por países como Honduras, Guatemala, y por Costa Rica que se encuentra posicionado en la casilla 62 . 


\section{Indice de Competitividad Global 2019}

El Foro Económico Mundial (WEF, por sus siglas en inglés) evaluó a 141 países en materia de competitividad para la elaboración del Índice Global de Competitividad 2019.

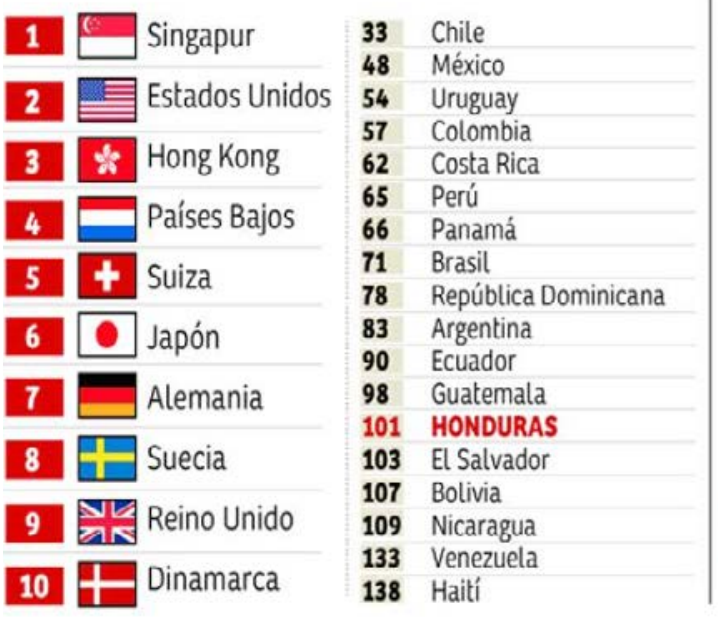

Doce pilares medidos en el estudio

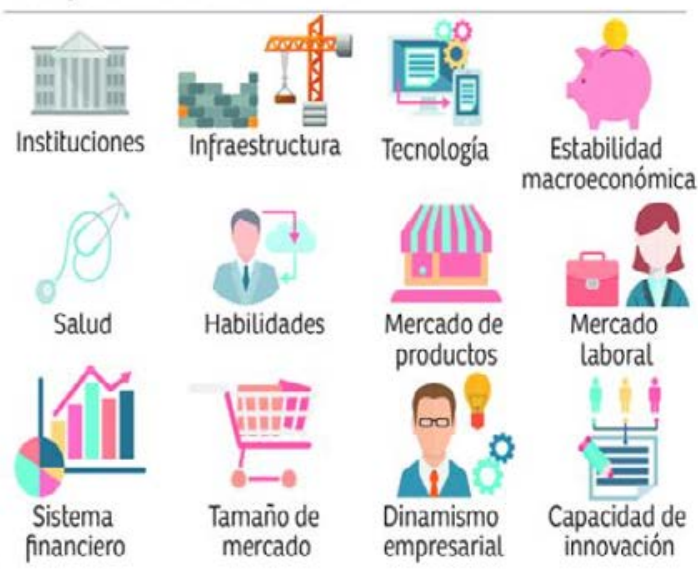

FUENTE INDICE DE COMPETTIMIDAD MUNDALL,

Figura 1 Tabla de índice de competitividad global 2019.

Es interesante que a pesar de estar ranqueados en una posición no muy bonancible en materia de innovación, El Salvador cuenta a partir del año 2019 con una Secretaría de Innovación de la Presidencia, la cual está a cargo de un Secretario, nombrado por el mismo Presidente de la República, a quien le compete velar por la planificación, coordinación y fomento de las estrategias de innovación del Estado, procurando el desarrollo de las capacidades de los servidores públicos, teniendo como resultado el mejoramiento de los servicios proporcionados por parte de la diferentes entidades del Órgano Ejecutivo.
¿Qué significa gerenciar la seguridad y salud en el trabajo?

$\mathrm{Al}$ conocer las expectativas en relación con la innovación, es importante hacer la relación con el término gerenciamiento que significa: cosas diferentes para personas diferentes. Algunos lo identifican con funciones realizadas por empresarios, gerentes o supervisores; otros, lo refieren a un grupo particular de personas (Gerencia y Negocos, s.f.). 
La gerencia moderna está sujeta a constantes actualizaciones de los marcos jurídicos y tendencias de las diversas instancias que inciden de una $\mathrm{u}$ otra forma en sus procesos productivos, inclusive más en aquellos aspectos que conciernen a sus recursos humanos que en la era de la globalización están fuertemente regidos por la salud integral y la prevención de los riesgos laborales bajo el concepto de salud ocupacional. La Seguridad y Salud en el Trabajo no se limita a cuidar las condiciones físicas y sicológicas de los trabajadores, o cuidar los bienes de las empresas; también supone un apoyo a su perfeccionamiento y al mantenimiento y optimización de su capacidad de trabajo, por ello busca controlar los accidentes y evitar las enfermedades ocupacionales mediante la reducción de las condiciones de riesgo que puedan provocar enfermedades y/o accidentes laborales.

Esto la convierte en una herramienta muy útil dentro de la cultura organizacional de hoy, identificada con la humanización del trabajo. Actualmente en El Salvador, son pocas las empresas que cuentan con departamentos bien estructurados en materia de seguridad y salud en el trabajo o un gerente o director en Seguridad y Salud Ocupacional. Por lo general estas áreas son vistas como la antesala de un eminente despido de la organización, ya que muchas organizaciones ubican en estos departamentos a colaboradores que están por jubilarse o mientras llega el retiro los colocan en estas áreas o a los que nadie quiere en sus operaciones les dan a manera de castigo la oportunidad de aportar «algo» en estas áreas.

Es por lo anterior que se vuelve necesaria la innovación en seguridad y salud ocupacional ya que los acontecimientos recientes han puesto en evidencia que si una empresa u organización quiere seguir manteniéndose operativa debe contar con personal competente en la materia. Otro aspecto importante para tomar en cuenta es la brecha generacional que todo gerente debe enfrentar actualmente, y donde se debe considerar que no es lo mismo trabajar con un colaborador que sea Milenial o Generación Y que con un Baby Boomer o incluso alguien de edad mayor a 66 años que es de la generación tradicional.

Ante el gerenciamiento de la Seguridad y Salud en el Trabajo estos cambios generacionales se han venido a incrementar ahora con la emergencia sanitaria que estamos viviendo, ya que el efecto de cuido y responsabilidad puede variar entre una generación y otra. 

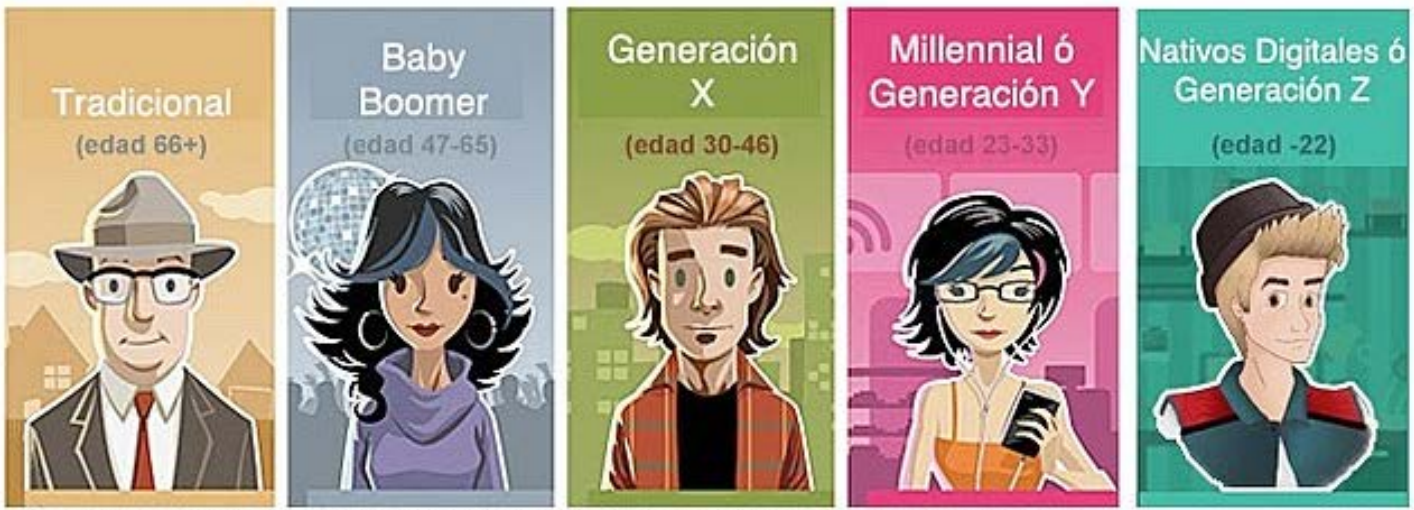

Figura 2 Detalle de brechas generacionales.

Si el nombre gerente o director es muy fuerte para las organizaciones, es recomendable designar otra denominación, sabemos que los títulos anteriores son sinónimos de salarios «jugosos», aunque la realidad en El Salvador estas áreas son vistas como males necesarios y difícilmente podrán justificar salarios atractivos que justifiquen la responsabilidad que conlleva la denominación de gerente o director en Seguridad y Salud Ocupacional. Sin embargo, es a principios del año 2020 en el que este rubro toma un giro y aquí si diremos que gracias al COVID-19 el área de seguridad y salud ocupacional ha pasado de ser un plato de segunda mesa a convertirse en el protagonista de la historia reciente.
El COVID-19, la enfermedad provocada por el nuevo coronavirus fue reportada por primera vez a finales del año 2019 en China y en pocos meses se convirtió en pandemia. El pasado lunes 28 de septiembre de 2020 la cantidad de muertes por la enfermedad llegó al hito sombrío del millón con América y El Caribe a la cabeza (BBC News Mundo, 2020).

Los empleadores tendrán la responsabilidad global de asegurarse de que se adopten todas las medidas de prevención y protección factibles para reducir al mínimo los riesgos profesionales ${ }^{1}$.

$1 \quad$ El artículo 16 del Convenio sobre seguridad y salud de los trabajadores, 1981 (núm. 155) dispone que: "Deberá exigirse a los empleadores que, en la medida en que sea razonable y factible, garanticen que los lugares de trabajo [...] que estén bajo su control son seguros y no entrañan riesgo alguno para la seguridad y la salud de los trabajadores". 
Los empleadores tienen la responsabilidad de suministrar, cuando sea necesario y en la medida en que sea razonable y factible, ropas y equipos de protección apropiados sin costo alguno para el trabajador ${ }^{2}$.

Reconociendo el gran reto que la economía mundial está enfrentando, el pasado 28 de abril de 2020, fecha en que se celebra a nivel internacional la seguridad y salud en el trabajo, se enfocó en abordar el brote de enfermedades infecciosas en el trabajo, prestando especial atención a la actual pandemia de COVID-19.

Las recientes experiencias con el síndrome respiratorio agudo severo (SRAS), la gripe A (H1N1) y los brotes del virus del Ébola han puesto de relieve la importancia de centrarse en los lugares de trabajo no solo para identificar a las poblaciones de riesgo, sino también para comprender los mecanismos de propagación de las enfermedades y aplicar medidas eficaces de control y prevención. Los lugares de trabajo son plataformas eficaces en las que los empleadores y los trabajadores pueden difundir información y sensibilizar sobre la SST, en particular sobre las medidas de prevención y protección para reducir la propagación de las enfermedades infecciosas (Organización Internacional del Trabajo (OIT), 2020).
A raíz de esta enfermedad la necesidad de contar y/o fortalecer los departamentos de Seguridad y Salud Ocupacional en las empresas es ahora uno de los pilares fundamentales para poder garantizar la continuidad de las operaciones de los negocios, el visibilizar la gestión de la prevención de riesgos ocupacionales es ahora en día un punto importante de las gestiones el cual si bien es cierto aún es imperativo contar con personas competentes en la materia que se encarguen de gestionar, liderar o gerenciar el tema de la Seguridad y Salud Ocupacional, las empresas que deseen seguir operando sin ningún problema deberán considerar este recurso en sus operaciones.

Estamos aún en pañales para decir que los niveles de prevención son maduros y se encuentran bien arraigados en las culturas organizacionales, pero bien o mal las autoridades estatales relacionadas en la materia, entiéndase el Ministerio de Trabajo y Previsión Social realizan prueba y error con intentos por supervisar el cumplimiento de las medidas preventivas en el tema de riesgos biológicos.

Gerenciamiento de la seguridad y salud en el trabajo en la modernidad líquida de Zygmunt Bauman

Hoy en día el estilo de vida que tenemos en nuestra sociedad es tan variable que no exis-

2 Artículos 16, 3, y 21 del Convenio núm. 155. 
te una seguridad plena en temas relacionados con el trabajo o las relaciones interpersonales, de acuerdo al sociólogo de origen polaco Zygmunt Bauman estamos viviendo una etapa conocida como modernidad liquida, y relacionado a los acontecimientos actuales de una pandemia, aspectos como el gerenciamiento de la seguridad y salud en el trabajo son temas que hasta unos meses eran relegados a un segundo plano, entendemos que el querer un ambiente estable en materia de seguridad y salud en el trabajo se convierte en un reto ambicioso para una persona que este liderando o gerenciando un departamento o área relacionada con la prevención de riesgos, sobre todo, porque el hecho de estar inmersos en una modernidad donde la estabilidad o solidez como le decía Bauman son más que pura utopía.

Al respecto, Bauman (2000) dice «Cuerpo y comunidad son los últimos puestos defensivos del casi abandonado campo de batalla donde cada día, con pocos respiros, se entabla la lucha por la seguridad, la certidumbre y la protección». Tomando como referencia lo planteado por Bauman, la sociedad líquida está en cambio constante, lo que genera una angustia existencial, donde parece no haber sentido cuando se trata de construir nuevas cosas, ya que el tiempo y la propia modernidad impulsarán su desintegración. Así nos encontramos como raza humana navegando los mares de la incertidumbre, sin saber cómo estará la economía mañana, si estallará una crisis o no, si contaremos con trabajo, si formaremos una familia, etc.
Los conflictos que la liquidez muestra en el mundo laboral

En su teoría, Bauman expone que uno de los hábitos más afectados por la modernidad líquida es el laboral. Las personas ya no ingresan a un trabajo sabiendo cuando se retirarán, sino que eso depende de las reglas del mercado y, por tanto, es incierto. Lo que hace más complicado el gerenciamiento en materia de seguridad y salud en el trabajo, sobre todo, porque las personas no harán un vínculo de lealtad con la empresa; por otro lado, la incertidumbre de su estabilidad laboral puede influir a que exista un mayor auge de accidentabilidad en las actividades de una organización.

Imaginemos el ambiente laboral de 50 años atrás donde una persona comenzaba a trabajar en una compañía en la que permanecía hasta retirarse; el mercado en el siglo XXI exige renovación y cambios desde la propia empresa, pero también desde las necesidades profesionales.

En el entorno post pandemia, las relaciones interpersonales se están viendo afectadas por la necesidad del distanciamiento físico entre las personas, y junto con las tendencias de la modernidad líquida, donde los roles sociales y las instituciones han cambiado para siempre. Ya nadie quiere comprometerse a largo plazo. Un empleo ya no es suficiente para crear una carrera. 


\section{Resultados}

De acuerdo a los datos publicados por la Universidad Johns Hopkins hasta el 24 de octubre de 2020, la pandemia de COVID-19, en el mundo lleva un total de 40,514,091 de casos confirmados, de los cuales $1,120,003$ personas han fallecido producto de esta enfermedad (Johns Hopkins University, 2020), lo que demuestra que es importante tomar las medidas necesarias de prevención en todo momento, para garantizar una continuidad de los negocios y evitar brotes epidémicos en las organizaciones, áreas de seguridad y salud en el trabajo que juegan un papel preponderante en la contención del avance de esta enfermedad y, sobre todo, buscar que la economía de los países no se vea más impactada por los cierres ordenados por las autoridades gubernamentales.

El gerenciamiento de la seguridad y salud en el trabajo post COVID-19 exige respuestas no convencionales, cuyo factor esencial sea el componente de la innovación para poder de esta manera lograr el objetivo que las organizaciones continúen operando sin dificultad.

¿Qué deberían hacer los empleadores durante un brote epidémico en sus centros de trabajo?

Todo empleador tendrá la responsabilidad de asegurarse de que se adopten las medidas de prevención y protección factibles para reducir al mínimo los riesgos profesionales (Convenio sobre Seguridad y Salud de los Trabajadores, $1981)^{3}$. Los empleadores tienen la responsabilidad de suministrar, cuando sea necesario y en la medida «en que sea razonable» y factible, ropas y equipos de protección apropiados sin costo alguno para el trabajador.

Los gerentes de Seguridad y Salud en el trabajo deben considerar en sus planeaciones estratégicas los siguientes inspecciones laborales, instrumentos esenciales para promover el cumplimiento de las normativas, protocolos, debiendo innovar en la forma en la que cumplen sus funciones, tanto de asesoramiento a empleadores y a trabajadores, como de la fiscalización, adoptando, de ser necesario, medidas de aplicación inmediata en los casos de incumplimiento grave o de peligro inminente para la salud de los trabajadores.

Esta crisis también está produciendo graves problemas de salud mental. Depresión por aislamiento, estrés por sobrecarga de trabajo, temor a perder emprendimientos, el trabajo e inseguridad respecto al futuro de las relaciones laborales, miedo al contagio, problemas de conciliación y agotamiento emocional podrán, entre otros factores, generar enfermedades mentales que podrían perdurar más allá de los efectos de la pandemia.

$3 \quad$ El artículo 16 del Convenio sobre seguridad y salud de los trabajadores, 1981 (núm. 155) dispone que: "Deberá exigirse a los empleadores que, en la medida en que sea razonable y factible, garanticen que los lugares de trabajo [...] que estén bajo su control son seguros y no entrañan riesgo alguno para la seguridad y la salud de los trabajadores". 
Además, las desigualdades de género se agudizan en este contexto: por ejemplo, las mujeres del sector de salud y cuidados representan un $70 \%$ del total (Organización Internacional del Trabajo, 2020) que enfrenta como consecuencia de la pandemia una doble carga: turnos más largos en el trabajo y el trabajo doméstico y de cuidado no remunerado en sus hogares, que luego de cierre de las instituciones educativas y de cuidado se ha visto aumentado de forma considerable. Cabe añadir la necesidad de una especial protección frente al riesgo de las trabajadoras embarazadas y en periodo de lactancia.

El acceso a vacaciones y licencias retribuidas ha sido una de las medidas adoptadas por los países para poder aplicar las medidas de aislamiento físico recomendadas por las autoridades. Por último, el contagio de las personas o de sus familiares no puede suponer ningún tipo de discriminación, exclusión o estigmatización, en particular en el mantenimiento o en la búsqueda de nuevos emprendimientos, empleo y en la posterior reinserción laboral en el caso de los trabajadores.

Finalmente, el diálogo social, a través de mecanismos como el funcionamiento de instancias nacionales tripartitas, la negociación colectiva y los comités de seguridad y salud en las empresas son elementos clave para construir acuerdos y encon- trar soluciones consensuadas en estas materias que puedan ser aplicadas de forma exitosa.

Las normas internacionales del trabajo relativas a la Seguridad y Salud en el Trabajo (SST) son un punto de referencia para revisar los derechos y deberes de los actores frente a la crisis provocada por el brote.

La crisis sanitaria que tanto El Salvador como el mundo entero está viviendo actualmente, es una oportunidad única para revalorizar la importancia de la seguridad y la salud laboral como una de las condiciones fundamentales para el trabajo decente, como señala en la Declaración Tripartita del Centenario de la OIT para el Futuro del Trabajo (2019).

Después de superada la crisis sanitaria será un aspecto clave a considerar en la activación productiva y en la recuperación económica con la finalidad de evitar nuevos brotes.

\section{Conclusiones}

Como parte de las conclusiones se reconoce que el COVID-19 dejará de ser una pandemia para convertirse en una endemia; es decir, una enfermedad con la que tendremos que convivir, así como en el caso del VIH/SIDA, la gripe común, entre otras, al menos hasta que la 
comunidad científica descubra una vacuna que brinde la inmunidad apropiada contra el virus.

Por otra parte, mientras eso no suceda, las empresas están haciendo lo posible porque la Seguridad y Salud en el trabajo se vuelva uno de los pilares fundamentales para continuar con sus operaciones y evitar un mayor deterioro de la economía, buscando colocar como primer punto la importancia de la seguridad y salud de las personas y luego la productividad de la empresa.

La innovación es el aliado para salir de la zona de confort que muchos gerentes o administradores en seguridad y salud en el trabajo deben procurar un mundo diferente. Por último, es necesario resaltar que después de superada la crisis sanitaria, la prevención de riesgos en los lugares de trabajo, será un aspecto clave a considerar en la activación productiva y en la recuperación económica.

\section{Referencias consultadas}

Bauman, Z. (2000). Modernidad líquida, Buenos Aires, Argentina: Fondo de Cultura Económica.

BBC News Mundo (29 de septiembre de 2020); Coronavirus: el mapa que muestra el número de infectados y muertos en el mundo por COVID-19. Obtenido de Coronavirus: https://www.bbc.com/mundo/noticias-54347255

Cuerpo de Bomberos de El Salvador. (2015). Boletín Especial, San Salvador
El Salvador.com (11 de octubre de 2019); EL Salvador.com. Obtenido Capacidad de Innovación frena la competitividad de El Salvador: https://www. elsalvador.com/eldiariodehoy/capacidad-de-innovacion-frena-la-competitividad-de-el-salvador/648374/2019

Gerencia y Negocios (s.f). Conceptos de Gerencia y Gestión. Obtenido de: https:/gerenciaynegocios.com/ canales/gerencia_gestion/conceptos_gerencia/

INCAE Bussiness School. (2016). Innovación: el reto más importante para la gerencia en este nuevo siglo. Obtenido de: https://www.incae.edu/es/ blog/2016/08/10/innovacion-el-reto-mas-importante-para-la-gerencia-en-este-nuevo-siglo.html

Johns Hopkins University. (2020). COVID-19 Dashboard by the Center for System Science and Engineering (CSSE). Obtenido de: https://www.arcgis.com/ apps/opsdashboard/index.html\#/bda7594740fd$40299423467 b 48$ e9ecf6.

Organización Internacional del Trabajo (OIT). (2020). Frente a la Pandemia: Garantizar la Seguridad y Salud en el Trabajo. Ginebra: Organización Internacional del Trabajo (OIT). Obtenido de https://www.ilo.org/ wcmsp5/groups/public/---ed_protect/---protrav/--safework/documents/publication/wcms_742732.pdf

Organización Internacional del Trabajo (OIT). (2020). Seguridad y salud en el trabajo frente a la pandemia; mesa virtual de dialogo de OIT America Latina. Lima: OIT. 
Guillermo Francisco Barrera Córdova

Innovación en el gerenciamiento de la seguridad y salud ocupacional post COVID-19

págs. 128 - 140

P.F. Drucker, La disciplina de la innovación, Harvard bussiness school publishing corporation, 1985. disponible en http://www.sela.org/media/3212238/r-la-disciplina-de-la-innovacion.pdf
J. Echavarría, Manual de Oslo y la Innovación Social. (2008) ARBOR Ciencia, Pensamiento y Cultura, vol. 184, no. 732, pp. 609-618, 2008. Doi: https://doi. org/10.3989/arbor. 2008. i732.210 